$$
\begin{aligned}
& \text { National Aeronautics } \\
& \text { and } \\
& \text { Space Administration }
\end{aligned}
$$

Grant NGR-39-007-025

Technical Report No. 1

\title{
EFFECT OF PLATE THICKNESS ON THE BENDING STRESS DISTRIBUTION AROUND THROUGH CRACKS
}

by

R. J.Hartranft and G. C. Sih

\author{
Department of Applied Mechanics \\ Lehigh University \\ Bethlehem, Pennsylvania
}

June, 1967 


\section{LIST OF SYMBOLS}

a $f^{c}(x, \alpha), f^{S}(x, \alpha)$ $\underset{t(\rho)}{g(\rho), h(\rho), s(\rho),}$

h

k

$m, n$

$r, \theta$

$u(y), v(y)$

w

$x, y, z$

$A(\alpha), B(\alpha), C(\alpha)$

D

E

$\mathrm{H}_{x y}$

$I_{0}, K_{0}$

$J_{0}, J_{1}$

$\mathrm{K}_{1}$

$M_{0}, M^{*}$

$M_{X}, M_{y}$

$R_{j}, \theta_{j} \quad(j=1,2)$

$v_{x}, v_{y}$

$\beta^{\beta}, \beta_{y}$

$\gamma$
Half crack length or bond length

Cosine and sine transforms of $f(x, y)$, respectively

Functions of $\rho$

Plate thickness

$h / \sqrt{10}$

Real parameters

Polar coordinates measured from origin

Unknowns

Transverse deflection of plate

Cartesian coordinates

Arbitrary functions in solution of Reissner's equations

Flexural rigidity

Young's modulus

Twisting stress couple

Modified Bessel functions of the first and second kinds, respectively

Bessel functions of the first kind

Moment intensity factor

Uniform and resultant moments, respectively

Bending stress couples

Polar coordinates measured from crack tips

Transverse shear stress resultants

Average rotations

Multiplying factor of $k_{1}$ for external cracks 


$\begin{array}{ll}\delta(\rho) & \text { Dirac delta generalized function } \\ \lambda & k / a \\ \nu & \text { Poisson's ratio } \\ \Phi(\xi), \Psi(\xi) & \text { Solutions of Fredholm integral equation }\end{array}$




\author{
AROUND THROUGH CRACKS \\ by \\ R.J.Hartranft ${ }^{2}$ and G. C. $\operatorname{Sin}^{3}$
}

$\underline{\text { ABSTRACT }}$

Using the plate bending theory developed by Reissner, this paper is concerned with the influence of plate thickness on the bending stress distribution near the singular crack point for two basic geometries. These are the internal or finite crack and the external or semi-infinite cracks in a plate of infinite extent. The boundary problems are formulated by means of Fourier sine or cosine transforms and then reduced to systems of dual integral equations. The behavior of the local stresses is governed by a single Fredholm integral equation of the second kind which can be solved numerically. It is found that the bending strengths of cracked plates generally decrease as the plate thickness increases from zero to some finite, but small, value. This is evidenced by the high elevation of the intensity of the local stresses as the ratio of plate thickness to crack (or bond) length is perturbed slightly from zero. In particular, moment-intensity factor, which governs the magnitude of the moment distribution around the crack tip, is defined. Such a factor has been known in fracture mechanics to determine the onset of rapid crack extension in brittle materials.

This investigation was carried out as part of a research program sponsored by the National Aeronautics and Space Administration under Grant NGR-39-007-025 with Lehigh University.

2 Assistant Professor of Mechanics, Lehigh University, Bethlehem, Pennsylvania.

3 Professor of Mechanics, Lehigh University, Bethlehem, Pennsylvania. 
One of the basic requirements for the strength analysis of plates containing flaws or cracks is the knowledge of the singular character of the stress field in the neighborhood of the crack point. Approximate, but useful, information may be conveniently assessed by assuming the material to behave elastically everywhere in the plate. When dealing with plate geometries of the discontinuous type such as the crack-like configuration, the mathematical problem involves the determination of solutions to certain differential equations with mixed boundary conditions. The solution of such mixed boundary-value problems is considerably more complicated than that of their unmixed counterparts.

In recent years, several investigators have discussed the nature of the local stresses around a sharp crack in a thin plate subjected to out-of-plane bending loads. Based on the PoissonKirchhoff theory of thin plates and the technique of Fadie eigenfunction expansions, williams $[1]^{4}$ found that the elastic stresses near the tip of a semi-infinite crack vary as the inverse square root of the radial distance from the crack front. His results were not complete in that the strength or magnitude of the local stresses was left undetermined. It was Sih and Rice [2,3] who later cleared the way for finding the coefficients in the eigenfunction expansions by application of the theory of complex functions. However, since the results in [1-3] were obtained from the ${ }^{4}$ Numbers in brackets designate References at end of paper. 
classical fourth order theory of thin plates, the edge conditions at the crack surfaces are satisfied only in an approximate manner in that the three physically natural boundary conditions of prescribing bending moment, twisting moment and transverse shear stress are replaced by two conditions. Owing to such a replacement, the stress distribution in the immediate neighborhood of the crack edges will naturally be affected and will not be accurate.

To overcome the aforementioned shortcoming, Knowles and Wang [4] employed the sixth-order theory of Reissner [5] and obtained the crack-tip stresses in a plate whose thickness is vanishingly sma11. Their ingenious analysis makes use of the theory of singular integral equations. However, the extension of the method in [4] to cracked plates with finite plate thickness does not appear to be tractable.

Presented in this paper is another method of solution which allows the examination of the effect of plate thickness on the detailed structure of the bending stresses in a region close to the crack front. Two basic problems are considered. In the first, an infinite plate with a straight crack of finite length is subjected to all around bending at infinity. A special case of this problem is that in [4] when the plate thickness becomes vanishing1y small. In the second, the infinite plate is weakened by two semi-infinite, collinear cracks and is bent symmetrically about the bond plane connecting the crack tips. Stress solutions are computed and plotted versus the ratio of plate thickness to crack 
or bond length.

\section{REISSNER'S EQUATIONS}

The detailed features of the Reissner theory of plate bending can be found in [5]. Hence, only those equations which are pertinent to the present discussion will be cited. With reference to a system of rectangular coordinates $x, y$, bending and twisting moments will be denoted by $M_{x}, M_{y}$ and $H_{x y}$ while $V_{x}$ and $V_{y}$ will stand for the transverse shear stress resultants. The transverse deflection of the midplane of the plate is $w$. In the absence of lateral loads on the faces of the plate, $w, V_{x}$, and $V_{y}$ are required to satisfy the differential equations

$$
\begin{aligned}
& \frac{\partial v_{x}}{\partial x}+\frac{\partial v_{y}}{\partial y}=0 \\
& v_{x}-k^{2} \nabla^{2} v_{x}=-D \frac{\partial}{\partial x}\left(\nabla^{2} w\right) \\
& v_{y}-k^{2} \nabla^{2} v_{y}=-D \frac{\partial}{\partial y}\left(\nabla^{2} w\right)
\end{aligned}
$$

in which $D=E h^{3} / 12\left(1-v^{2}\right)$ is the flexural rigidity of the plate with E being the Young's modulus, $h$ the plate thickness, and $v$ the Poisson's ratio. The Laplacian operator $\nabla^{2}$ in two-dimensions is $\partial^{2} / \partial x^{2}+\partial^{2} / \partial y^{2}$ and $k^{2}=h^{2} / 10$. Once $w, V_{x}$, and $V_{y}$ are known, the moments may be found from

$$
M_{x}=-D\left(\frac{\partial^{2} w}{\partial x^{2}}+v \frac{\partial^{2} w}{\partial y^{2}}\right)+2 k^{2} \frac{\partial v_{x}}{\partial x}
$$




$$
\begin{aligned}
& M_{y}=-D\left(\frac{\partial^{2} w}{\partial y^{2}}+v \frac{\partial^{2} w}{\partial x^{2}}\right)+2 k^{2} \frac{\partial V_{y}}{\partial y} \\
& H_{x y}=-(1-v) D \frac{\partial^{2} w}{\partial x \partial y}+k^{2}\left(\frac{\partial V_{x}}{\partial y}+\frac{\partial V_{y}}{\partial x}\right)
\end{aligned}
$$

and the average rotations $\beta_{x}, \beta_{y}$ about the $y$ - and $x$-axes are given by

$$
\begin{aligned}
& \beta_{x}=-\frac{\partial W}{\partial x}+\frac{2 k^{2}}{(1-v) D} v_{x} \\
& \beta_{y}=-\frac{\partial W}{\partial y}+\frac{2 k^{2}}{(1-v) D} v_{y}
\end{aligned}
$$

In the sequel, the midplane of the plate will always occupy the $x y-p l a n e$ with the $z$-axis being normal to the plate surface. On a typical edge of the plate, say $x=0$, it is possible to specify one member of each of the pairs

$$
\left(M_{x}, \beta_{x}\right) \quad\left(H_{x y}, \beta_{y}\right) \quad\left(V_{x}, w\right)
$$

Moreover, if the problem possesses symmetry about the $x z-p l a n e$, the conditions

$$
\beta_{y}=H_{x y}=V_{y}=0 \text {, for } y=0
$$

must be fulfilled.

\section{FORMULATION OF THE PHYSICAL PROBLEM}

Let an infinite plate of constant thickness $h$ be cut along certain straight segments belonging to the yz-plane and be subjected to bending loads in such a way that the problem is sym- 


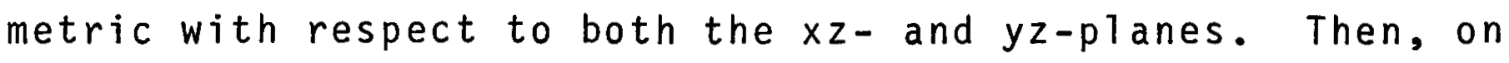
the uncut portion of the plate in the yz-plane, the symmetry conditions

$$
\beta_{x}=H_{x y}=V_{x}=0 \text {, for } x=0
$$

may be enforced. Since the edges of the cut are to be free from twisting moment and transverse shear, the last two conditions apply in the entire yz-plane, i.e.,

$$
H_{x y}(0, y)=V_{x}(0, y)=0
$$

for all values of $y$.

Now, the $y$-dependence in eqs. (1.1) through (3.2) may be removed by subjecting all these equations to either the Fourier sine or cosine transform in $y$ according to whether the function transformed is odd or even in $y$. Assumed to be satisfied are the regularity conditions such as that the displacements, etc. are bounded as $x^{2}+y^{2} \rightarrow \infty$. In this way, the resulting ordinary differential equations may be solved without difficulty. Applying the symmetry conditions, eq. (5), the solution in the transformed domain may be written as

$$
\begin{aligned}
\alpha^{2} D W^{C}= & e^{-\alpha x} A(\alpha)[\alpha x+B(\alpha)] \\
\alpha(1-v) D \beta_{x}^{C} & =e^{-\alpha x}\left\{A(\alpha)\left[(1-v)(\alpha x+B(\alpha)-1)-4 k^{2} \alpha^{2}\right]\right. \\
& \left.+4 k^{2} \alpha^{2} p(x, \alpha) C(\alpha)\right\}
\end{aligned}
$$




$$
\begin{aligned}
\alpha(1-v) D \beta_{y}^{5} & =e^{-\alpha x}\left\{A(\alpha)\left[(1-v)(\alpha x+B(\alpha))-4 k^{2} \alpha^{2}\right]\right. \\
& \left.+4 k^{2} \alpha^{2} s(\alpha k) p(x, \alpha) C(\alpha)\right\}
\end{aligned}
$$

and

$$
\begin{aligned}
M_{x}^{C} & =e^{-\alpha x}\left\{A(\alpha)\left[2+4 k^{2} \alpha^{2}-(1-\nu)(\alpha x+B(\alpha))\right]\right. \\
& \left.-4 k^{2} \alpha^{2} s(\alpha k) p(x, \alpha) C(\alpha)\right\} \\
M_{y}^{C} & =e^{-\alpha x}\left\{A(\alpha)\left[2 \nu-4 k^{2} \alpha^{2}+(1-v)(\alpha x+B(\alpha))\right]\right. \\
& \left.+4 k^{2} \alpha^{2} s(\alpha k) p(x, \alpha) C(\alpha)\right\} \\
H_{x y}^{S} & =e^{-\alpha x}\left\{A(\alpha)\left[4 k^{2} \alpha^{2}-(1-v)(\alpha x+B(\alpha)-1)\right]\right. \\
& \left.-2\left(1+2 k^{2} \alpha^{2}\right) p(x, \alpha) C(\alpha)\right\} \\
V_{x}^{C} & =2 \alpha e^{-\alpha x}\{p(x, \alpha) C(\alpha)-A(\alpha)\} \\
V_{y}^{S} & =2 \alpha e^{-\alpha x}\{s(\alpha k) p(x, \alpha) C(\alpha)-A(\alpha)\}
\end{aligned}
$$

in which

$$
s(p)=\left[1+\left(1 / \rho^{2}\right)\right]^{\frac{1}{2}}, p(x, \alpha)=\exp \{\alpha x[1-s(\alpha k)]\}
$$

The superscripts $c$ and $s$ are introduced to denote, respectively, the cosine and sine transforms of the function under consideration. For instance,

$$
w^{c}(x, \alpha)=\int_{0}^{\infty} w(x, y) \cos \alpha y d y
$$


is the cosine transform of the deflection $w(x, y)$, and

$$
\beta_{y}^{S}(x, \alpha)=\int_{0}^{\infty} \beta_{y}(x, y) \sin \alpha y d y
$$

is the sine transform of the average rotation $\beta_{y}(x, y)$. Once the unknown functions $A(\alpha), B(\alpha)$ and $C(\alpha)$ are determined in the transformed domain, the appropriate inversions, i.e., for example,

$$
\begin{aligned}
& w(x, y)=\frac{2}{\pi} \int_{0}^{\infty} w^{c}(x, \alpha) \cos \alpha y d \alpha \\
& \beta_{y}(x, y)=\frac{2}{\pi} \int_{0}^{\infty} \beta_{y}^{S}(x, \alpha) \sin \alpha y d \alpha
\end{aligned}
$$

may be used to convert the solution into the physical domain.

Taking the transform of eq. (6), i.e.,

$$
H_{X y}^{S}(0, \alpha)=V_{X}^{C}(0, \alpha)=0
$$

and applying these conditions to eqs. $(8.3)$ and $(8.4)$ yield

$$
A(\alpha)=C(\alpha), B(\alpha)=-(1+v) /(1-v)
$$

Hence, eqs. (7.1) to (8.5) may be expressed in terms of a single unknown $A(\alpha)$ as

$$
\begin{aligned}
& \alpha^{2} D W^{C}=e^{-\alpha x} A(\alpha)\{\alpha x-[(1+v) /(1-v)]\} \\
& \alpha(1-v) D B_{x}^{C}=e^{-\alpha x} A(\alpha)\left\{(1-v) \alpha x-2-4 k^{2} \alpha^{2}[1-p(x, \alpha)]\right\}
\end{aligned}
$$




$$
\begin{aligned}
\alpha(1-\nu) D \beta_{y}^{S} & =e^{-\alpha x} A(\alpha)\{(1-\nu) \alpha x-(1+\nu) \\
& \left.-4 k^{2} \alpha^{2}[1-s(\alpha k) p(x, \alpha)]\right\}
\end{aligned}
$$

and

$$
\begin{aligned}
M_{x}^{c}= & e^{-\alpha x} A(\alpha)\{3+v-(1-v) \alpha x \\
+ & \left.4 k^{2} \alpha^{2}[1-s(\alpha k) p(x, \alpha)]\right\} \\
M_{y}^{c}= & -e^{-\alpha x} A(\alpha)\{(1-v)(1-\alpha x) \\
& \left.+4 k^{2} \alpha^{2}[1-s(\alpha k) p(x, \alpha)]\right\} \\
H_{x y}^{S} & e^{-\alpha x} A(\alpha)\left\{-(1-v) \alpha x+\left(2+4 k^{2} \alpha^{2}\right)[1-p(x, \alpha)]\right\} \\
V_{x}^{c}= & 2 \alpha e^{-\alpha x} A(\alpha)[p(x, \alpha)-1] \\
V_{y}^{S}= & 2 \alpha e^{-\alpha x} A(\alpha)[s(\alpha k) p(x, \alpha)-1]
\end{aligned}
$$

The function $A(\alpha)$ can be evaluated from the single remaining boundary condition of specifying either $M_{x}$ or $\beta_{x}$ on the edge $x=0$. This will be done in the subsequent sections.

\section{SINGLE INTERNAL CRACK}

Suppose that a thin plate containing a crack of length $2 a$, Fig. 1, is bent by uniform moment of intensity $M_{0}$ at infinity. The solution to this problem may be obtained by judiciously superposing the simple solution of an uncracked plate under uniform bending to that of a cracked plate with bending moment applied to the crack surfaces. Thus, it suffices to solve the 
problem of specifying uniform moment $M_{0}$ on the segment $x=0$, $|y|<a$ of the plate. Referring to Fig. 1, the conditions to be satisfied on $x=0$ are

$$
\begin{aligned}
& B_{x}(0, y)=0,|y|>a \\
& M_{x}(0, y)=M_{0},|y|<a
\end{aligned}
$$

Because of symmetry with respect to the $x z-p l a n e$, it can be easily deduced from eqs. (9.2) and $(10.1)$ that $A_{1}(\alpha)$ satisfies the pair of dual integral equations

$$
\begin{aligned}
& \int_{0}^{\infty} \frac{1}{\alpha} A_{1}(\alpha) \cos \alpha y d \alpha=0, y>a \\
& \int_{0}^{\infty} A_{1}(\alpha) t(\alpha k) \cos \alpha y d \alpha=\frac{\pi}{2} M_{0}, y<a
\end{aligned}
$$

where the function $t(\alpha k)$ stands for

$$
t(\rho)=3+v+4 \rho^{2}[1-s(\rho)]
$$

The objective here is to reduce the simultaneous system of dual integral equations to a single integral equation involving one unknown function. This can be accomplished by first defining

$$
u(y)=\frac{2}{\pi} \int_{0}^{\infty} \frac{1}{\alpha} A_{1}(\alpha) \cos \alpha y d \alpha, y<a
$$

In view of eq. (12.1), the Fourier inversion theorem [6] gives 


$$
\frac{1}{\alpha} A_{1}(\alpha)=\int_{0}^{a} u(y) \cos \alpha y d \alpha
$$

Equation (14) may be substituted into the integrated expression of eq. (12.2) and the problem reduces to the determination of the function $u(y)$ such that ${ }^{5}$

$$
\int_{0}^{\infty} t(\alpha k) \sin \alpha y d \alpha \int_{0}^{a} u(n) \cos \alpha n d n=\frac{\pi}{2} M_{0} y, y<a
$$

and

$$
u(y)=0, y>a
$$

As a departure from the method of solution employed in [4], the determination of the behavior of $u(y)$ near the endpoints of the crack will be somewhat guided by the solution of the analogous crack problem in classical plate theory. In the classical theory, the singular character of the stresses arises from the solution

$$
u^{*}(y)=\frac{M_{0}}{3+v} \sqrt{a^{2}-y^{2}}, y<a
$$

Therefore, it is plausible to admit in the modified theory the representation

$$
u(y)=\int_{y}^{a} \frac{\psi(t) t d t}{\sqrt{t^{2}-y^{2}}}, y<a
$$

${ }^{5}$ If the order of integration were reversed, eq. (15.1) would become a singular integral equation as in [4]. 
so that in the limit as $y \rightarrow a, u(y)$ becomes

$$
u(y)=\psi(a) \sqrt{a^{2}-y^{2}}+0\left(a^{2}-y^{2}\right)^{3 / 2}
$$

where the leading term preserves the $y$-dependence of the classical solution, $u^{*}(y)$. Note that the representation, eq. (16), is a special case of Abel's integral equation [7] whose solution is

$$
\psi(t)=-\frac{2}{\pi} \int_{t}^{a} \frac{u^{\prime}(y) d y}{\sqrt{y^{2}-t^{2}}}, t<a
$$

Upon substitution of eq. (16) in (15.1) and use of the identity $[8]$

$$
\int_{0}^{t} \frac{\cos \alpha y}{\sqrt{t^{2}-y^{2}}} d y=J_{0}(\alpha t)
$$

where $J_{0}$ is the usual zero-order Bessel function of the first kind, eq. $(15.1)$ becomes

$$
\int_{0}^{a} \psi(t) t d t \int_{0}^{\infty} t(\alpha k) J_{0}(\alpha t) \sin \alpha y d \alpha=M_{0} y, y<a
$$

Now, let

$$
g(\rho)=\frac{1}{1+v}\left\{2+4 \rho^{2}[1-s(\rho)]\right\}=0\left(\frac{1}{\rho^{2}}\right) \text {, as } \rho \rightarrow \infty
$$

such that $t(p)$ may be expressed as

$$
t(p)=(1+v)[1+g(p)]
$$


Making use of eq. (20) and the known result [8]

$$
\int_{0}^{\infty} J_{0}(\alpha t) \sin \alpha y d \alpha=\left\{\begin{array}{c}
0, y<t \\
\left(y^{2}-t^{2}\right)-\frac{1}{2}, y>t
\end{array}\right.
$$

it is possible to put eq. (19) in the form

$$
\begin{aligned}
\int_{0}^{y} \frac{\psi(t) t d t}{\sqrt{y^{2}-t^{2}}} & =\frac{M_{0}}{1+v} y-\int_{0}^{a} \psi(t) t d t \int_{0}^{\infty} g(\alpha k) J_{0}(\alpha t) \sin \alpha y d \alpha \\
y & <a
\end{aligned}
$$

which coincides with a special case of Abel's integral equation. From a relationship similar to that between eqs. (16) and (18), eq. (21) can be transformed to

$$
\begin{aligned}
\psi(t) & +\int_{0}^{a} \psi(\tau) \tau d \tau \int_{0}^{\infty} \alpha g(\alpha k) J_{0}(\alpha \tau) J_{0}(\alpha t) d \alpha \\
& =\frac{M_{0}}{1+v}, t<a
\end{aligned}
$$

Introducing the dimensionless quantities

$$
\lambda=\frac{k}{a}, \xi=\frac{t}{a}, \Phi(\xi)=\frac{1+v}{M_{0}} \sqrt{\xi} \psi(a \xi)
$$

the Fredholm integral equation

$$
\Phi(\xi)+\int_{0}^{1} F(\xi, n) \Phi(n) d n=\sqrt{\xi}, \xi<1
$$

is obtained. The kernel $F(\xi, n)$ is given by 


$$
F(\xi, n)=\sqrt{\xi \eta} \int_{0}^{\infty} s g(\lambda s) J_{0}(\xi s) J_{0}(n s) d s
$$

Another form of $F(\xi, \eta)$ that is more amenable to numerical calculation is

$$
\begin{aligned}
F(\xi, n) & =\frac{C}{\lambda^{2}} \sqrt{\xi \eta} I_{0}\left(\xi \frac{n}{\lambda}\right) K_{0}\left(n \frac{n}{\lambda}\right) \\
& -\sqrt{\xi \eta} \int_{0}^{\infty} s f(\lambda s) J_{0}(\xi s) J_{0}(n s) d s, 0<\xi \leq n
\end{aligned}
$$

since the function

$$
f(\rho)=\frac{C}{\rho^{2}+n^{2}}-g(\rho)=0\left(\frac{1}{\rho^{6}}\right) \text {, as } \rho \rightarrow \infty
$$

where $C=[2(1+v)]^{-1}, n^{2}=1 / 2$ and hence the integral in eq. (24) converges rapidly. In eq. (24), $I_{0}$ and $K_{0}$ are the modified Bessel functions of the first and second kinds, respectively.

It is clear that $\Phi(\xi)$ fully determines the solution of the problem. From eq. (16), the function $u(y)$ may be found:

$$
\begin{aligned}
u(y) & =\frac{M_{0} a^{2}}{1+v} \int_{y / a} \frac{\sqrt{\xi} \Phi(\xi) d \xi}{\sqrt{a^{2} \xi^{2}-y^{2}}} \\
& =\frac{M_{0}}{1+v}\left\{\Phi(1) \sqrt{a^{2}-y^{2}}-\int_{y / a}^{1} \sqrt{a^{2} \xi^{2}-y^{2}}\left[\frac{\Phi(\xi)}{\sqrt{\xi}}\right]^{\prime} d \xi\right\}
\end{aligned}
$$

Inserting eq. (25) into eq. (14), the only unknown in eqs. (9.1) through (10.5) is obtained: 


$$
\begin{aligned}
A_{1}(\alpha) & =\frac{\pi M_{0} a^{2} \alpha}{2(1+\nu)} \int_{0}^{1} \sqrt{\xi} \Phi(\xi) J_{0}(\alpha a \xi) d \xi \\
& =\frac{\pi M_{0} a}{2(1+\nu)}\left\{\Phi(1) J_{1}(\alpha a)-\int_{0}^{1} J_{1}(\alpha a \xi)\left[\frac{\Phi(\xi)}{\xi}\right] \xi d \xi\right\}
\end{aligned}
$$

The solution of the problem has now been taken to a point at which the moments, shear resultants, etc. in the plate may be computed once $\Phi(\xi)$ is evaluated from eq. (23). Such a task can be best performed on an electronic computer. The numerical values of $\Phi(\xi)$ will be reported 1 ater on.

It should be pointed out that the convergence of the integral in eq. (24) becomes increasingly slower as $\lambda$ takes on smaller values. However, in the limit as $\lambda \rightarrow 0$, the results simplify considerably since

$$
g(\lambda s) \rightarrow \frac{2}{1+\nu}, F(\xi, n) \rightarrow \frac{2}{1+\nu} \delta(\xi-n) \text {, for } \lambda \rightarrow 0
$$

where $\delta(\rho)$ is the Dirac delta generalized function. Thus, for vanishingly thin plates, the classical results

$$
\begin{gathered}
\Phi(\xi)=\left(\frac{1+v}{3+v}\right) \sqrt{\xi}, u(y)=\frac{M_{0}}{3+v} \sqrt{a^{2}-y^{2}}, \\
A_{1}(\alpha)=\frac{\pi M_{0} a}{2(3+v)} J_{1}(\alpha a)
\end{gathered}
$$

are recovered.

\section{TWO EXTERNAL CRACKS}

The problem of two semi-infinite plates bonded together along 
a straight line segment of length 2 a as shown in Fig. 2 may be solved in the same way. The unbonded portions of the joined plates may be viewed as two semi-infinite cracks. To preserve a finite bending moment across the bond line described by $x=0$, $|y|<a$, the moment at infinity must fall to zero. The conditions for $x=0$ are

$$
\begin{aligned}
& \beta_{x}(0, y)=0,|y|<a \\
& M_{x}(0, y)=0,|y|>a
\end{aligned}
$$

For the present formulation, it is more convenient to express the condition, eq。 $(28,1)$, as

$$
\frac{\partial \beta_{x}(0, y)}{\partial y}=0,|y|<a
$$

In addition, the resultant moment $M^{\star}$ transmitted to the bond line from each half of the plate must be specified, i.e.,

$$
\int_{0}^{a} M_{x}(0, y) d y=\frac{1}{2} M^{*}
$$

By application of eqs. $(9.2)$ and $(10.1)$, the conditions expressed by eqs. $(28.2)$ and (29) become

$$
\begin{aligned}
& \int_{0}^{\infty} A_{2}(\alpha) t(\alpha k) \cos \alpha y d \alpha=0, y>a \\
& \int_{0}^{\infty} A_{2}(\alpha) \sin \alpha y d \alpha=0, y<a
\end{aligned}
$$


Similarly, eq. (30) takes the form

$$
\frac{2}{\pi} \int_{0}^{a} d y \int_{0}^{\infty} A_{2}(\alpha) t(\alpha k) \cos \alpha y d \alpha=\frac{1}{2} M^{*}
$$

From the pair of dual integral equations given by eqs. $(31.1)$ and $(31.2)$, subject to the condition eq. (32), the unknown function $A_{2}(\alpha)$ is fully determined.

Proceeding as in the problem of a single crack, a new function

$$
V(y)=\frac{2}{\pi} \int_{0}^{\infty} A_{2}(\alpha) t(\alpha k) \cos \alpha y d \alpha
$$

is defined such that the Fourier inversion theorem yields

$$
A_{2}(\alpha) t(\alpha k)=\int_{0}^{a} v(y) \cos \alpha y d y
$$

because $v(y)=0$ for $y>a$ as demanded by eq. (31.1). It follows that eq. (32) may be simplified:

$$
\int_{0}^{a} v(y) d y=A_{2}(0) t(0)=\frac{1}{2} M^{*}
$$

Eliminating the function $A_{2}(\alpha)$ in eq. (31.2), the unknown in the problem changes to $v(y)$ governed by

$$
\int_{0}^{\infty} \frac{\sin \alpha y}{t(\alpha k)} d \alpha \int_{0}^{a} v(n) \cos \alpha n d n=0, y<a
$$

and 


$$
v(y)=0, y>a
$$

To restrict the nature of the moment singularities at the crack tips, $v(y)$ is taken as

$$
v(y)= \begin{cases}0 & y>a \\ \frac{\gamma M^{*}}{\pi}\left[\frac{1}{\sqrt{a^{2}-y^{2}}}+\int_{y}^{a} \frac{\phi(t) d t}{\sqrt{t^{2}-y^{2}}}\right], 0<y<a\end{cases}
$$

which satisfies eq。( 34.2$)$ automatically. Before the remaining conditions can be expressed in terms of $\phi(t)$, it is necessary to calculate

$$
\begin{aligned}
A_{2}(\alpha) t(\alpha k) & =\int_{0}^{a} v(y) \cos \alpha y d y \\
& =\frac{\gamma M^{*}}{\pi}\left[\int_{0}^{a} \frac{\cos \alpha y}{\sqrt{a^{2}-y^{2}}} d y+\int_{0}^{a} \phi(t) d t \int_{0}^{t} \frac{\cos \alpha y}{\sqrt{t^{2}-y^{2}}} d y\right] \\
& =\frac{\gamma M^{*}}{2}\left[J_{0}(\alpha a)+\int_{0}^{a} \phi(t) J_{0}(\alpha t) d t\right]
\end{aligned}
$$

Putting $\alpha=0$ in eq. (36) and inserting the result subsequently into eq. (33) give

$$
\gamma=\left[1+\int_{0}^{a} \phi(t) d t\right]^{-1}
$$

Under these considerations, eq. (34.1) further reduces to 


$$
\begin{gathered}
\int_{0}^{\infty} \frac{J_{0}(\alpha a)}{t(\alpha k)} \sin \alpha a d \alpha+\int_{0}^{a} \phi(t) d t \int_{0}^{\infty} \frac{J_{0}(\alpha t)}{t(\alpha k)} \sin \alpha y d \alpha=0, \\
y<a
\end{gathered}
$$

The above integral equation may now be transformed to a special case of Abel's integral equation by introducing the function

$$
\begin{aligned}
h(\rho)= & 1-\frac{1+v}{t(\rho)}=\left(\frac{2}{3+v}\right) \frac{s(\rho)-1}{s(\rho)-[(1-v) /(3+v)]}=0\left(\frac{1}{\rho^{2}}\right), \\
& \text { as } \rho \rightarrow \infty
\end{aligned}
$$

into eq. (38). The result is

$$
\begin{aligned}
\int_{0}^{y} \frac{\phi(t) d t}{\sqrt{y^{2}-t^{2}}} & =\int_{0}^{\infty} h(\alpha k) J_{0}(\alpha a) \sin \alpha y d \alpha \\
& +\int_{0}^{a} \phi(t) d t \int_{0}^{\infty} h(\alpha k) J_{0}(\alpha t) \sin \alpha y d \alpha, y<a
\end{aligned}
$$

from which $\phi(t)$ is obtained [7]:

$$
\phi(t)=G_{1}(t, a)+\int_{0}^{a} G_{1}(t, \tau) \phi(\tau) d \tau, t<a
$$

where

$$
G_{1}(t, \tau)=t \int_{0}^{\infty} \alpha h(\alpha k) J_{0}(\alpha t) J_{0}(\alpha \tau) d \alpha
$$

The dimensionless notation

$$
\Psi(\xi)=\frac{a}{\sqrt{\xi}} \phi(a \xi)
$$


carries eq. (39) into

$$
\Psi(\xi)=G(\xi, 1)+\int_{0}^{1} G(\xi, n) \Psi(n) d n, \xi<1
$$

in which

$$
G(\xi, n)=\sqrt{\xi n} \int_{0}^{\infty} s h(\lambda s) J_{0}(\xi s) J_{0}(n s) d s
$$

When $\xi$ and $n$ are not zero simultaneously, the integrand in eq. (41) is $0\left(1 / s^{3 / 2}\right)$ as $s \rightarrow \infty$ and hence the integral converges. On the other hand, if both $\xi$ and $n$ are zero, the integrand is $0(1 / 5)$ as $s \rightarrow \infty$ and the integral diverges. For the purpose of finding the singular part of the integral in subsequent work and increasing the rate of convergence of the numerical evaluation of eq. (41), the identity

$$
\int_{0}^{\infty} \frac{s}{s^{2}+m^{2}} J_{0}(\xi s) J_{0}(n s) d s=I_{0}(\xi m) k_{0}(n m), 0<\xi \leq n
$$

will be used to put the kernel $G(\xi, n)$ in the form

$$
\begin{aligned}
G(\xi, n) & =\frac{C}{\lambda^{2}} \sqrt{\xi \eta} I_{0}\left(\xi \frac{m}{\lambda}\right) K_{0}\left(n \frac{m}{\lambda}\right) \\
& -\sqrt{\xi n} \int_{0}^{\infty} s q(\lambda s) J_{0}(\xi s) J_{0}(n s) d s, 0<\xi \leq n
\end{aligned}
$$

where $C=[2(1+v)]^{-1}$ as before and $m^{2}=(2+v) /[2(1+v)]$. The function $q(p)$ is obtained by comparing the Laurent series expansions of $h(\rho)$ and $c /\left(\rho^{2}+m^{2}\right)$, i.e., 
$q(\rho)=\frac{C}{\rho^{2}+m^{2}}-h(\rho)=0\left(\frac{1}{\rho^{\sigma}}\right)$, as $\rho \rightarrow \infty$

In terms of the solution, $\psi(\xi)$, of the Fredholm equation, eq. (35..2) gives

$$
v(y)=\frac{\gamma M^{*}}{\pi}\left[\frac{1}{\sqrt{a^{2}-y^{2}}}+\int_{y / a}^{1} \frac{\sqrt{\xi} \psi(\xi) d \xi}{\sqrt{a^{2} \xi^{2}-y^{2}}}\right], y<a
$$

and $A_{2}(\alpha)$ is obtainable from eq. (36) as

$$
A_{2}(\alpha)=\frac{\gamma M^{*}}{2 t(\alpha k)}\left[J_{0}(\alpha a)+\int_{0}^{1} \sqrt{\xi} \psi(\xi) J_{0}(\alpha a \xi) d \xi\right]
$$

in which $\gamma$ is

$$
\gamma=\left[1+\int_{0}^{1} \sqrt{\xi} \Psi(\xi) d \xi\right]^{-1}
$$

The problem is essentially solved since $\Psi(\xi)$ can be found numerically. Once this is done, the stresses and displacements everywhere in the plate may be calculated.

Some insight into the solution for small values of $\lambda$ can be gained by considering the limiting case of thin plates. When $\lambda \rightarrow 0$, it is not difficult to show that

$$
h(\lambda s) \rightarrow \frac{2}{3+\nu}, G(\xi, n) \rightarrow \frac{2}{3+\nu} \delta(\xi-n) \text {, for } \lambda \rightarrow 0
$$

In this case, the solution of eq. (40) is 


$$
\Psi(\xi)=\frac{2}{1+\nu} \operatorname{Lim}_{\varepsilon \rightarrow 0} \delta(\xi-1+\varepsilon)
$$

With $\gamma=(1+v) /(3+v)$, the functions $v(y)$ and $A_{2}(\alpha)$ simplify to

$$
v(y)=\frac{M^{*}}{\pi \sqrt{a^{2}-y^{2}}}, A_{2}(\alpha)=\frac{M^{*}}{2(3+v)} J_{0}(\alpha a)
$$

\section{STRESS DISTRIBUTION NEAR SINGULAR CRACK POINTS}

The distribution of stress in the vicinity of a sharp crack plays an important role in the examination of the stability behavior of plates weakened by flaws. Without going into the details of the theory of brittle fracture, which can be found in the work of Irwin [9], it is pertinent to find the singular part of the solution.

First, consider the problem of a plate with a finite crack under uniform bending, Fig. 1. From eq。(26), it is seen that the singular part of the solution depends only on the first term for $A_{1}(\alpha)$, i.e.,

$$
A_{1}(a)=\frac{\pi M_{0} a}{2(1+v)} \Phi(1) J_{1}(\alpha a)+\ldots
$$

Moreover, the functions $s(\rho)$ and $p(x, \alpha)$ in eqs. $(8.1)$ to $(8.5)$ can be expanded in Laurent series to give

$$
s(\rho)=1+\frac{1}{2} \frac{1}{\rho^{2}}-\frac{1}{8} \frac{1}{\rho^{3}}+\frac{1}{16} \frac{1}{\rho^{6}}+0\left(\frac{1}{\rho^{8}}\right) \text {, as } \rho \rightarrow \infty
$$




$$
\begin{aligned}
p(x, \alpha) & =1-\frac{x}{2 k^{2}} \frac{1}{\alpha}+\frac{x^{2}}{8 k^{4}} \frac{1}{\alpha^{2}}-\frac{x}{48 k^{4}}\left[\left(\frac{x}{k}\right)^{2}-6\right] \frac{1}{\alpha^{3}} \\
& +\frac{x^{2}}{384 k^{6}}\left[\left(\frac{x}{k}\right)^{2}-24\right] \frac{1}{\alpha^{4}}+0\left(\frac{1}{\alpha^{5}}\right) \text {, as } \alpha \rightarrow \infty
\end{aligned}
$$

Under these considerations, the transforms of the moments and shear resultants in eqs. (8.1) to (8.5) become

$$
\begin{aligned}
M_{x}^{c} & =\frac{\pi M_{0}^{a}}{2(1+v)} \Phi(1) e^{-\alpha x} J_{1}(\alpha a)[(1+v) \alpha x+(1+v) \\
& \left.-\frac{x^{2}}{2 k^{2}}+0\left(\frac{1}{\alpha}\right)\right] \\
M_{y}^{c} & =-\frac{\pi M_{0} a}{2(1+v)} \Phi(1) e^{-\alpha x} J_{1}(\alpha a)[(1+v) \alpha x-(1+v) \\
& \left.-\frac{x^{2}}{2 k^{2}}+0\left(\frac{1}{\alpha}\right)\right] \\
H_{x y}^{S} & =\frac{\pi M_{0} a}{2(1+v)} \Phi(1) e^{-\alpha x} J_{1}(\alpha a)\left[(1+v) \alpha x-\frac{x^{2}}{2 k^{2}}+0\left(\frac{1}{\alpha}\right)\right] \\
V_{x}^{c} & =-\frac{\pi M_{0} a}{1+\nu} \Phi(1) \cdot \alpha e^{-\alpha x} J_{1}(\alpha a)\left[\frac{x}{2 k^{2}} \frac{1}{\alpha}+0\left(\frac{1}{\alpha^{2}}\right)\right] \\
V_{y}^{s} & =-\frac{\pi M_{0} a}{1+v} \Phi(1) \cdot \alpha e^{-\alpha x} J_{1}(\alpha a)\left[\frac{x}{2 k^{2}} \frac{1}{\alpha}+0\left(\frac{1}{\alpha^{2}}\right)\right]
\end{aligned}
$$

Defining the polar coordinates $r, R_{1}, R_{2}, \theta_{1}, \theta_{1}, \theta_{2}$ as shown in Fig. 1, and making use of the Bessel integral-identities

$$
\int_{0}^{\infty} \alpha e^{-\alpha x} J_{1}(\alpha a) \cdot\left[\cos _{\sin }^{\sin } \alpha y d \alpha=-\frac{a}{\left(R_{1} R_{2}\right)^{3 / 2}}\left[\sin _{2}^{\cos } \frac{3}{2}\left(\theta_{1}+\theta_{2}\right)\right.\right.
$$


etc., eqs. (47.1) to (47.5) can be easily inverted into the physical domain:

$$
\begin{aligned}
M_{x}= & -\frac{\Phi(1) M_{0} a}{\sqrt{R_{1} R_{2}}}\left[-\cos \left(\frac{\theta_{1}+\theta_{2}}{2}-\theta\right)\right. \\
& \left.+\frac{a x}{R_{1} R_{2}} \sin \frac{3}{2}\left(\theta_{1}+\theta_{2}\right)\right]+0(1) \\
M_{y}= & -\frac{\Phi(1) M_{0} a}{\sqrt{R_{1} R_{2}}}\left[\frac{r}{a} \cos \left(\frac{\theta_{1}+\theta_{2}}{2}-\theta\right)\right. \\
& \left.-\frac{a x}{R_{1} R_{2}} \sin \frac{3}{2}\left(\theta_{1}+\theta_{2}\right)\right]+0(1) \\
H_{x y}= & -\frac{\Phi(1) M_{0} a}{\sqrt{R_{1} R_{2}}}\left[\frac{a x}{R_{1} R_{2}} \cos \frac{3}{2}\left(\theta_{1}+\theta_{2}\right)\right]+0(1) \\
V_{x}= & V_{y}=0(1) \text { as } R_{1} R_{2} \rightarrow 0
\end{aligned}
$$

It is useful to obtain explicitly the singular character of the moments near a crack tip, say $(0, a)$. As $R_{2} \rightarrow 0$, eqs。(48.1) to $(48.4)$ further reduce to

$$
\begin{aligned}
& M_{x}=-\frac{K_{1}}{\sqrt{2 R_{2}}}\left[\cos \frac{\theta_{2}}{2}+\frac{1}{2} \sin \theta_{2} \sin \frac{3}{2} \theta_{2}\right]+0(1) \\
& M_{y}=-\frac{K_{1}}{\sqrt{2 R_{2}}}\left[\cos \frac{\theta_{2}}{2}-\frac{1}{2} \sin \theta_{2} \sin \frac{3}{2} \theta_{2}\right]+0(1)
\end{aligned}
$$




$$
\begin{aligned}
& H_{x y}=-\frac{K_{1}}{\sqrt{2 R_{2}}} \cdot\left[\frac{1}{2} \sin \theta_{2} \cos \frac{3}{2} \theta_{2}\right]+0(1) \\
& v_{x}=v_{y}=0(1) \text {, as } R_{2} \rightarrow 0
\end{aligned}
$$

in which the shear resultants remain finite at the crack tip. This is in contrast to the classical solution [1] where $V_{x}=V_{y}$ $=0\left(R^{-3 / 2}\right)$ as $R_{2} \rightarrow 0$. For the special case of vanishingly thin plates, eq. (27) gives

$$
K_{1}=\frac{1+v}{3+v} M_{0} \sqrt{a}
$$

and eqs. (49) agree with those obtained by knowles and Wang [4].

In general, the parameter $K_{j}$, which controls the intensity of the local stress field, depends on the plate thickness $h$ and Poisson's ratio $v$ through $\Phi(1)$ as

$$
K_{1}=\Phi(1) M_{0} \sqrt{a}
$$

whose critical value has been known to determine the onset of rapid crack propagation [9]. The variation of $\Phi(1)$ with $\lambda=h /(a \sqrt{10})$ is computed numerically by solving the Fredholm integral equation given by eq. (23). The results are shown graphically in Fig. 3 for Poisson's ratio of $0.0,0.3$, and 0.5 . It is seen that the strength of the moment singularity, governed by $k_{1}$, increases rapidly as the plate thickness changes from zero to some finite, but small, value. For $v=0.3$ and a plate thick- 
ness to crack length ratio of 0.1 , the value of $k_{1}$ is approximately $62 \%$ greater than that found for a vanishingly thin plate. In fact, it can be demonstrated analytically that the rate of increase of the $k_{1}$-factor is infinite at $\lambda=0$, i.e.,

$$
\frac{\partial \Phi(1)}{\partial \lambda} \rightarrow \infty \text { as } \lambda \rightarrow 0
$$

The required proof is worked out in the Appendix.

An assumption of the Reissner theory of plate bending is that the in-plane stresses $\sigma_{x}, \sigma_{y}$ and ${ }^{\tau} x y$ vary linearly over the thickness of the plate:

$$
\left|\begin{array}{c}
\sigma_{x} \\
\sigma_{y} \\
\tau_{x y}
\end{array}\right|=\frac{12 z}{h^{3}}\left[\begin{array}{c}
M_{x} \\
M_{y} \\
H_{x y}
\end{array}\right]
$$

For this reason, some caution must be exercised in the application of the results in Fig. 3 when $\lambda$ becomes sufficiently large, since nonlinear distributions, especially local disturbances near the crack edges and the surfaces of the plate, are not accounted for in the present theory. However, further refinements would lead to a three-dimensional analysis of the problem which is the subject of another investigation.

The singular behavior of the solution for the problem of two semi-infinite plates joined along a finite segment, Fig. 2 , can 
be deduced in the same way. From eq. (44), $A_{2}(\alpha)$ may be approximated by

$$
A_{2}(\alpha)=\frac{\gamma M^{*}}{2} \frac{J_{0}(\alpha a)}{t(\alpha k)}+\cdots
$$

The Laurent expansions developed earlier for $s(\rho)$ and $p(x, \alpha)$ may again be used to obtain expressions of $M_{x}^{c}$, $M_{y}^{c}$, etc. which are similar in form to those in eqs. (47) except that they are now expressed in terms of the zero-order Bessel function, $J_{0}(\alpha a)$. The integrals required for the Fourier inversion are of the type

$$
\begin{aligned}
& \int_{0}^{\infty} \alpha \mathrm{e}^{-\alpha x} \mathrm{~J}_{0}(\alpha a)\left[{ }_{\cos }^{\sin }\right] \alpha y d \alpha \\
& =-\frac{r}{\left(R_{1} R_{2}\right)^{3 / 2}}\left[\cos _{\sin }^{\sin }\left[\theta-\frac{3}{2}\left(\theta_{1}+\theta_{2}\right)\right]\right.
\end{aligned}
$$

and so on. As before, the singular terms are found to be

$$
\begin{aligned}
M_{x} & =\frac{r M^{*}}{\pi \sqrt{R_{1} R_{2}}}\left\{\sin \left(\frac{\theta_{1}+\theta_{2}}{2}\right)-\frac{x r}{R_{1} R_{2}} \cos \left[\theta-\frac{3}{2}\left(\theta_{1}+\theta_{2}\right)\right]\right\} \\
& +0(1) \\
M_{y} & =\frac{r M^{*}}{\pi \sqrt{R_{1} R_{2}}}\left\{\sin \left(\frac{\theta_{1}+\theta_{2}}{2}\right)+\frac{x r}{R_{1} R_{2}} \cos \left[\theta-\frac{3}{2}\left(\theta_{1}+\theta_{2}\right)\right]\right\} \\
& +0(1)
\end{aligned}
$$




$$
\begin{aligned}
& H_{x y}=-\frac{\gamma M^{*}}{\pi \sqrt{R_{1} R_{2}}}\left\{\frac{x r}{R_{1} R_{2}} \sin \left[\theta-\frac{3}{2}\left(\theta_{1}+\theta_{2}\right)\right]\right\}+0(1) \\
& V_{x}=V_{y}=0(1) \text {, as } R_{1} R_{2} \rightarrow 0
\end{aligned}
$$

In the immediate vicinity of the crack tip at $(0,-a)$, i。e., as $R_{1} \rightarrow 0$, eqs. (53) can be simplified:

$$
\begin{aligned}
& M_{x}=\frac{K_{1}}{\sqrt{2 R_{1}}}\left[\cos \frac{\theta_{1}}{2}+\frac{1}{2} \sin \theta_{1} \sin \frac{3}{2} \theta_{1}\right]+0(1) \\
& M_{y}=\frac{K_{1}}{\sqrt{2 R_{1}}}\left[\cos \frac{\theta_{1}}{2}-\frac{1}{2} \sin \theta_{1} \sin \frac{3}{2} \theta_{1}\right]+0(1) \\
& H_{x y}=\frac{K_{1}}{\sqrt{2 R_{1}}}\left[\frac{1}{2} \sin \theta_{1} \cos \frac{3}{2} \theta_{1}\right]+0(1) \\
& V_{x}=V_{y}=0(1) \text { as } R_{1} \rightarrow 0
\end{aligned}
$$

Here, $k_{1}$ is defined as

$$
K_{1}=\frac{\gamma M^{*}}{\pi \sqrt{a}}
$$

Unlike eq. (50), the value of $K_{1}$ is inversely proportional to the square root of the only characteristic dimension in the plane of the plate. Note that, however, the angular variation of the singular part of the moments is the same as that shown in eqs. (49) for the finite crack problem. 
To find the variation of $\gamma$ in eq. (55) with $\lambda=h /(a \sqrt{10})$ where a is the half bond length, the integral equation, eq. (40), is first solved numerically on an electronic computer for $\psi(\xi)$ from which $\gamma$ is calculated by means of eq. (45). Figure 4 indicates how the slope of the curve becomes unbounded as $\lambda \rightarrow 0$, i.e.

$$
\frac{\partial \gamma}{\partial \lambda} \rightarrow 0, \text { as } \lambda \rightarrow 0
$$

This condition is verified in the Appendix. The significance of this result is that the local intensification of the bending stresses increases appreciably as the plate thickness is varied slightly from zero. For example, as $h$ increases from zero to one-tenth the bond length, $K_{1}$ increases approximately $62 \%$ for $\nu=0.3$. The point on the curve in Fig. 4 for $\lambda=0$ corresponds to $\gamma=(1+v) /(3+v)$.

Finally, it should be mentioned that the present method of solution can also be applied to solve boundary problems of cracked plates owing to skew-symmetric bending loads such as twisting moments. The same type of results would be expected.

\section{APPENDIX}

The proof that the slopes of the curves in Figs. 3 and 4 become infinite at $\lambda=0$ will now be given. To this end, it suffices to show that the expressions $\partial \Phi(1) / \partial \lambda$ and $\partial \gamma / \partial \lambda$ become unbounded in the limit as $\lambda \rightarrow 0$. 
Differentiating the Fredholm integral equation given by eq. (23) with respect to $\lambda$ gives

$$
\frac{\partial \Phi(\xi)}{\partial \lambda}+\int_{0}^{1}\left[\frac{\partial F(\xi, n)}{\partial \lambda} \Phi(n)+F(\xi, n) \frac{\partial \Phi(n)}{\partial \lambda}\right] d n=0, \xi<1
$$

in which

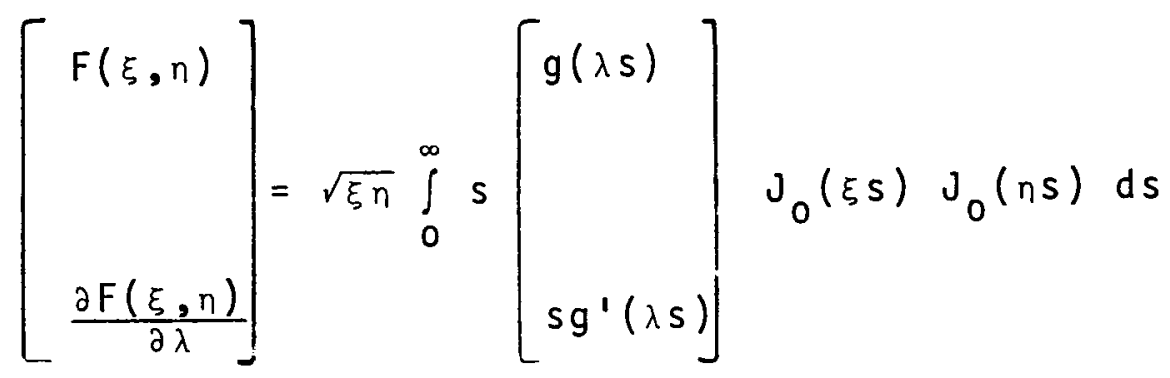

As $\lambda \rightarrow 0$, it can be easily established that

$$
g(\lambda s) \rightarrow \frac{2}{1+\nu}, g^{\prime}(\lambda s) \rightarrow-\frac{4}{1+\nu}, \Phi(\xi) \rightarrow \frac{1+\nu}{3+\nu} \sqrt{\xi}
$$

and hence

$$
\begin{gathered}
\lim _{\lambda \rightarrow 0} \int_{0}^{1} \frac{\partial F(\xi, n)}{\partial \lambda} \Phi(n) d n=-\frac{4 \sqrt{\xi}}{3+v} \int_{0}^{\infty} s^{2} J_{0}(\xi s) d s \int_{0}^{1} n J_{0}(n s) d n \\
\quad=-\frac{4 \sqrt{\xi}}{3+\nu} \int_{0}^{\infty} s J_{0}(\xi s) J_{j}(s) d s
\end{gathered}
$$

Remembering that

$$
F(\xi, n) \rightarrow \frac{2}{1+v} \delta(\xi-n) \text {, as } \lambda \rightarrow 0
$$


and setting $\xi=1$, eq。(57) yields the result

$$
\lim _{\lambda \rightarrow 0} \frac{\partial \Phi(1)}{\partial \lambda}=\frac{4(1+v)}{(3+\nu)^{2}} \int_{0}^{\infty} s J_{0}(s) J_{1}(s) d s
$$

Since the integrand may be written as

$$
\begin{aligned}
& s J_{0}(s) J_{1}(s)=\frac{1}{\pi}\left[-\cos 2 s+\frac{1}{4 s}(2+\sin 2 s)\right. \\
& \left.\quad+0\left(\frac{1}{s^{2}}\right)\right] \text { as } s \rightarrow \infty
\end{aligned}
$$

the integral in eq. (58) becomes unbounded at the upper limit and thus eq. (51) is verified.

To establish eq. (56), the same line of reasoning may be followed. Starting from eq. (40) in the form

$$
\begin{aligned}
\frac{\partial \Psi(\xi)}{\partial \lambda} & =\frac{\partial G(\xi, 1)}{\partial \lambda}+\int_{0}^{1}\left[\frac{\partial G(\xi, n)}{\partial \lambda} \Psi(n)+G(\xi, n) \frac{\partial \Psi(n)}{\partial \lambda}\right] d n, \\
& \xi<1
\end{aligned}
$$

with

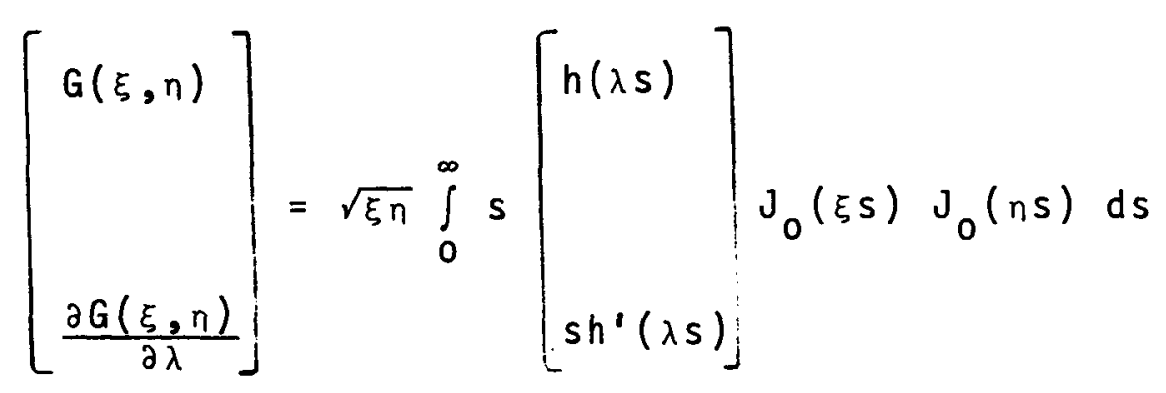


it can be shown that

$$
\begin{aligned}
& \lim _{\lambda \rightarrow 0} \frac{\partial \Psi(\xi)}{\partial \lambda}=\frac{\partial G(\xi, 1)}{\partial \lambda}+\frac{2}{3+\nu} \lim _{\lambda \rightarrow 0} \frac{\partial \Psi(\xi)}{\partial \lambda} \\
& +\frac{2}{1+\nu} \int_{0}^{1} \frac{\partial G(\xi, n)}{\partial \lambda}\left[\lim _{\varepsilon \rightarrow 0} \delta(n-1+\varepsilon)\right] d n
\end{aligned}
$$

since

$$
G(\xi, n) \rightarrow \frac{2}{3+\nu} \delta(\xi-\eta) \text { as } \lambda \rightarrow 0
$$

and

$$
h(0)=\frac{2}{3+v}, h^{\prime}(0)=-\frac{4(1+v)}{(3+v)^{2}}
$$

Equation (60) may be simplified and rearranged to read as

$$
\frac{1+\nu}{3+v} \lim _{\lambda \rightarrow 0} \frac{\partial \Psi(\xi)}{\partial \lambda}=\frac{3+v}{1+v} \frac{\partial G(\xi, 1)}{\partial \lambda}
$$

and hence it follows from eq. (45) that

$$
\lim _{\lambda \rightarrow 0} \frac{\partial \gamma}{\partial \lambda}=-\int_{0}^{1} \sqrt{\xi} \frac{\partial G(\xi, 1)}{\partial \lambda} d \xi
$$

where the integrand is given by

$$
\sqrt{\xi} G(\xi, 1)=-\frac{4(1+v)}{(3+v)^{2}} \xi \int_{0}^{\infty} s^{2} J_{0}(\xi s) J_{0}(s) d s
$$

Carrying out the integration yields 


$$
\begin{aligned}
\lim _{\lambda \rightarrow 0} \frac{\partial \gamma}{\partial \lambda} & =\frac{4(1+v)}{(3+v)^{2}} \int_{0}^{1} \xi d \xi \int_{0}^{\infty} s^{2} J_{0}(\xi s) J_{0}(s) d s \\
& =\frac{4(1+v)}{(3+v)^{2}} \int_{0}^{\infty} s J_{0}(s) J_{1}(s) d s \rightarrow \infty
\end{aligned}
$$

which is the same as eq. (58). This completes the proof of eq. (56).

\section{REFERENCES}

1. Williams, M. L., "The Bending Stress Distribution at the Base of a Stationary Crack", J.Appl. Mech., Vol。28, (1961), p. 78 .

2. Sih, G. C. and Rice, J. R., "The Bending of Plates of Dissimilar Materials with Cracks", J. Appl. Mech., Vol. 31, (1964), p. 477 .

3. Sih, G. C. and Rice, J.R., Authors' Closure of Discussion of "The Bending of Plates of Dissimilar Materials with Cracks", J. App1. Mech., Vol. 32, (1965), p. 464.

4. Knowles, J. K. and Wang, N. M., "On the Bending of an Elastic Plate Containing a Crack", J. Math. and Phys., Vol. 39, (1960), p. 223。

5. Reissner, E., "The Effect of Transverse Shear Deformation on the Bending of Elastic Plates", J. Appl. Mech., Vol. 12, (1945), p. A-69.

6. Sneddon, I. N., Fourier Transforms, McGraw-Hi11, New York, 1951 .

7. Whittaker, E. T. and Watson, G. N., Modern Analysis, Fourth Edition, Cambridge, 1962.

8. Watson, G. N., Theory of Bessel Functions, Second Edition, Cambridge, 1958 .

9. Irwin, G. R., "Analysis of Stresses and Strains Near the End of a Crack Tŕaversing a Plate", J. Appl. Mech., Vol. 24, (1957), p. 361 . 


\section{TITLE OF FIGURES}

FIGURE 1 - FINITE CRACK IN INFINITE PLATE

FIGURE 2 - SEMI-INFINITE CRACKS IN INFINITE PLATE

FIGURE 3 - MOMENT INTENSITY VERSUS RATIO OF PLATE THICKNESS TO HALF CRACK LENGTH

FIGURE 4 - MOMENT INTENSITY VERSUS RATIO OF PLATE THICKNESS TO HALF BOND LENGTH 


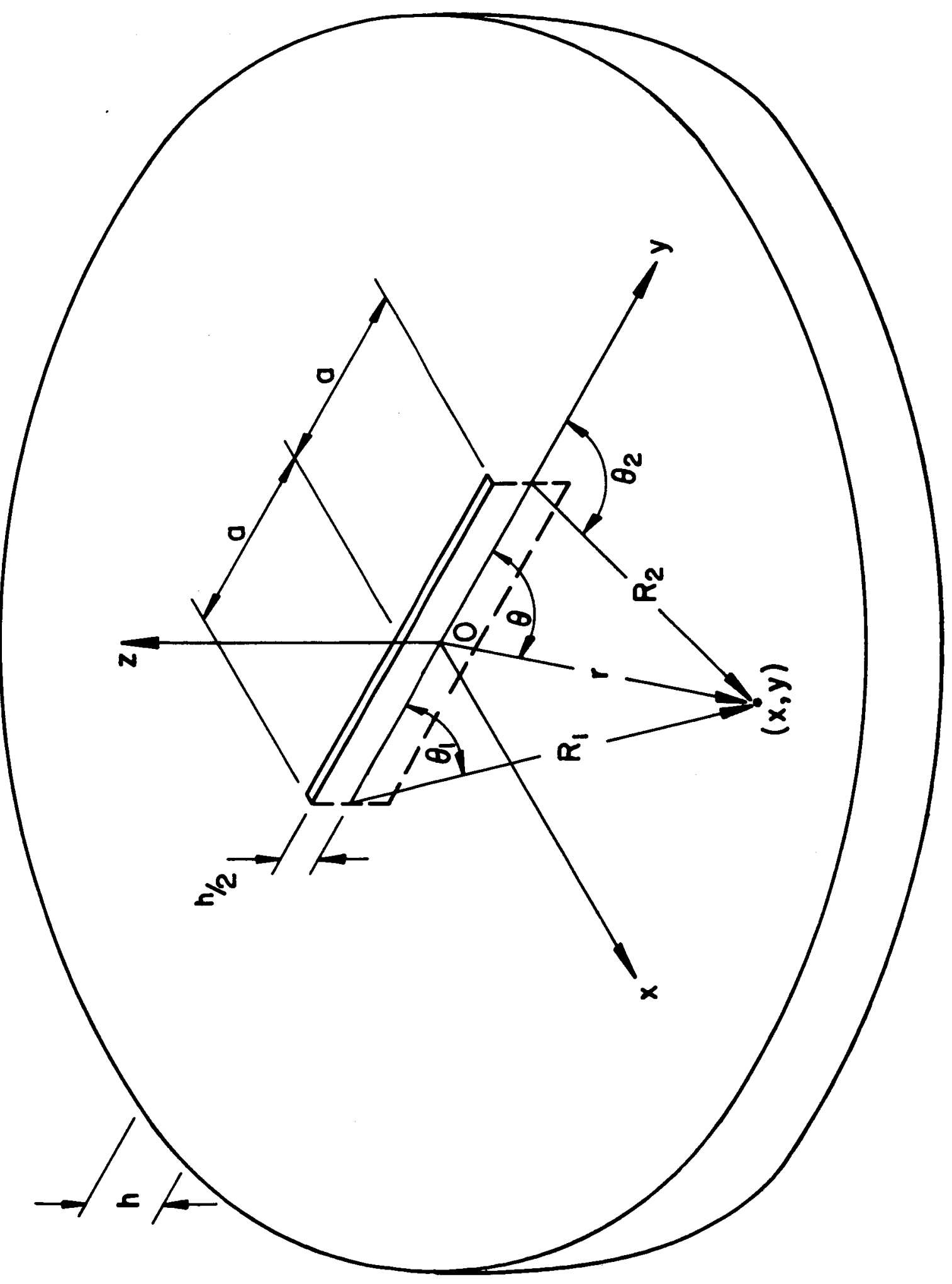




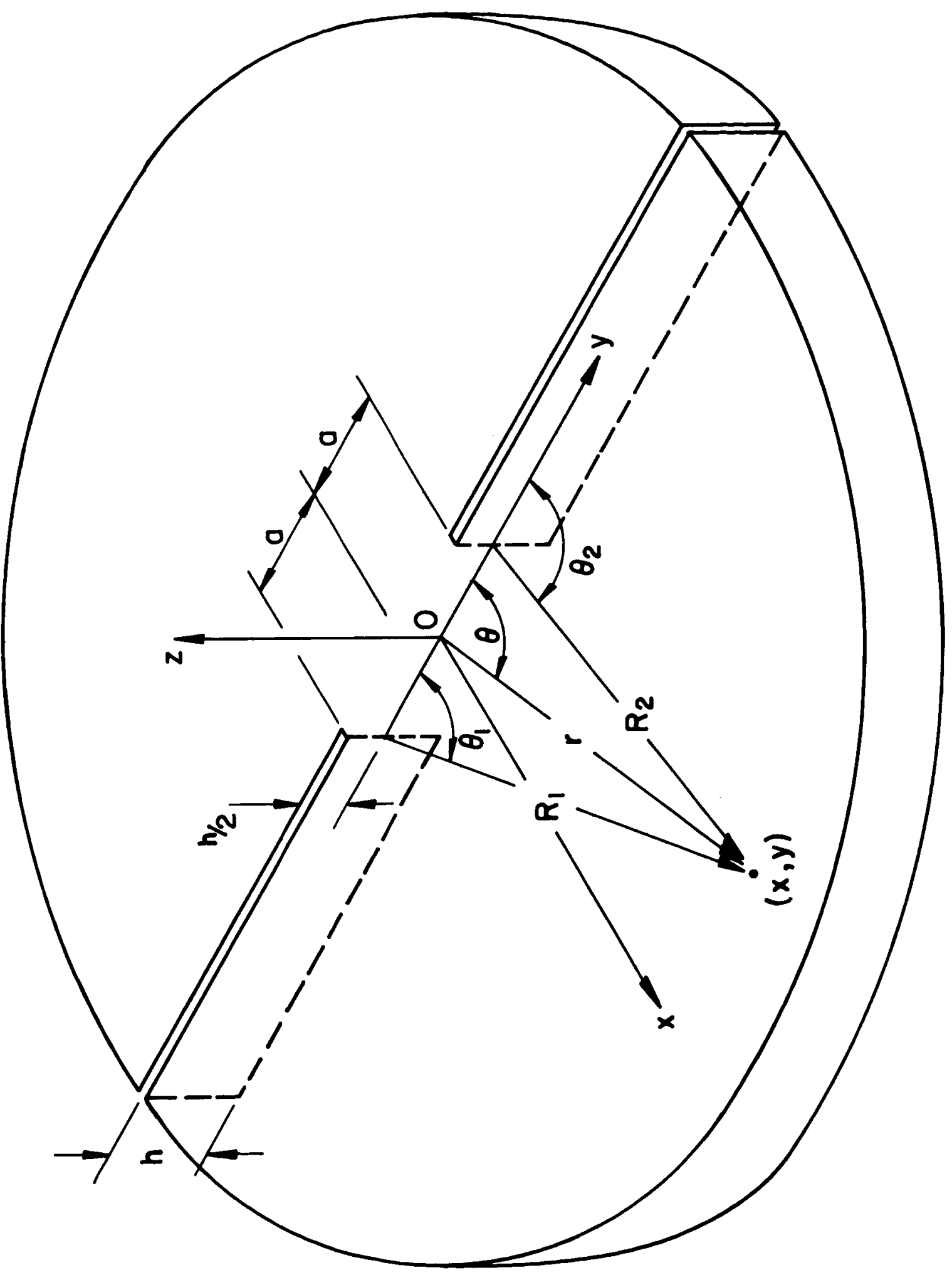




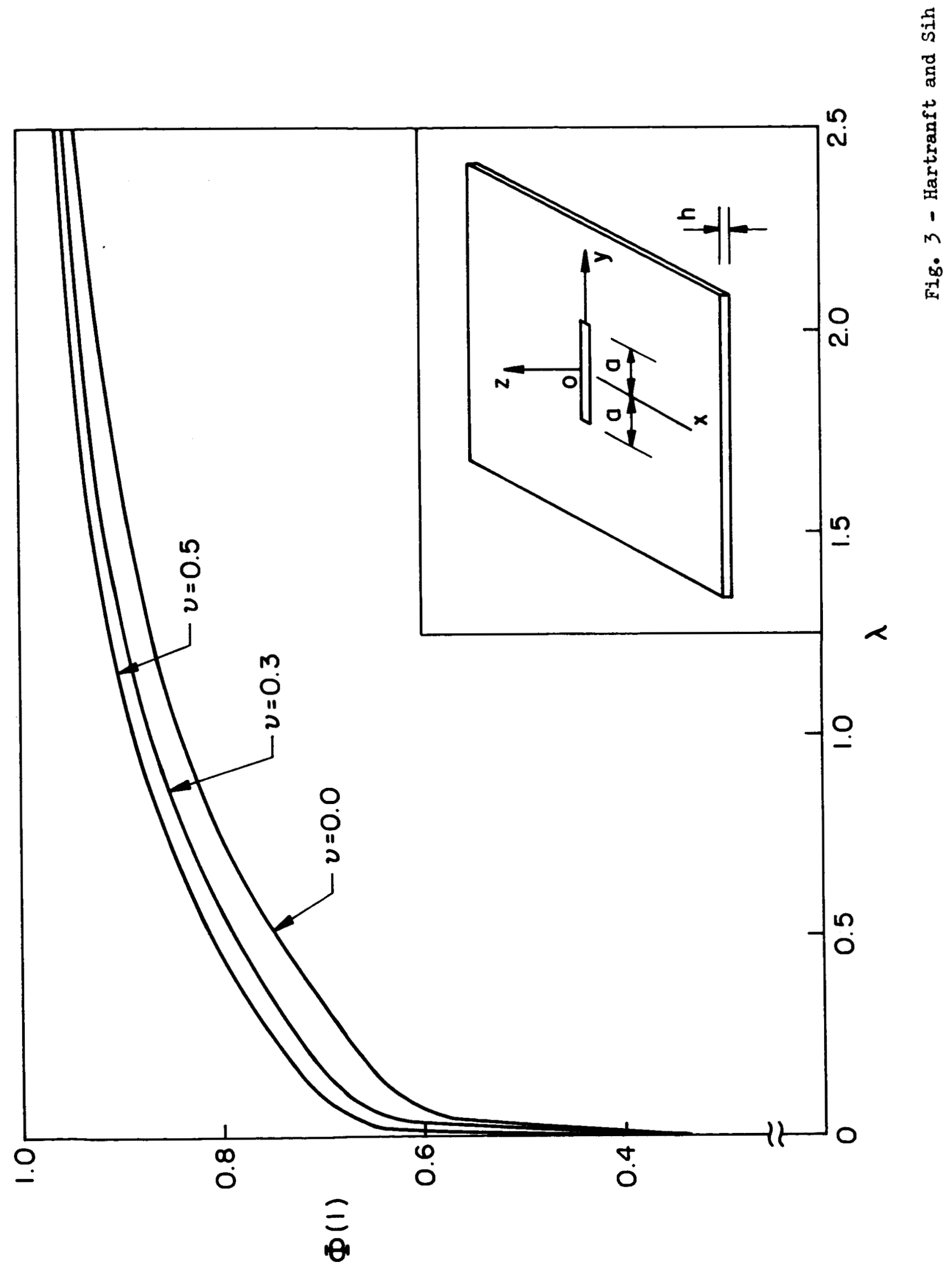




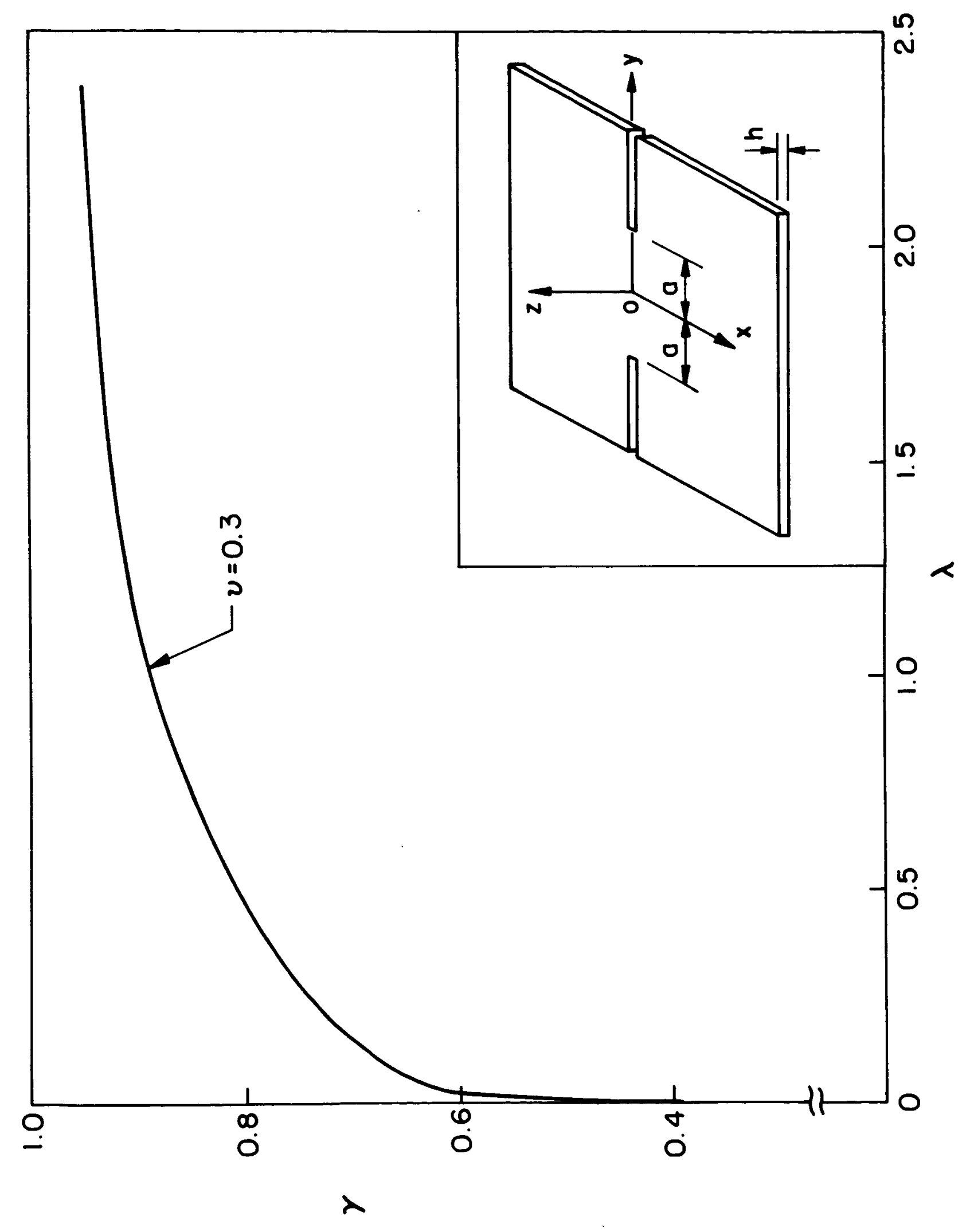

